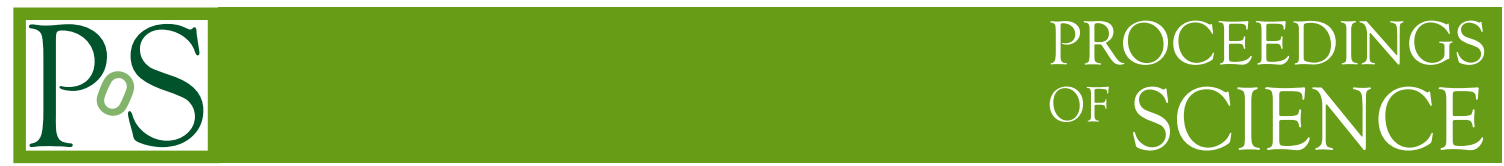

\title{
Operation of the CMS Silicon Tracker
}

\section{Derek A. Strom*i}

University of Illinois at Chicago

E-mail: derek.axel.stromecern.ch

The CMS tracker is the largest silicon detector ever constructed, covering 200 square meters and providing an average of 14 high-precision measurements per track. The use of tracker data for reconstruction of charged particles and primary and secondary vertices requires fine-grained monitoring and calibration procedures as well as accurate alignment. Results from timing and threshold optimization, gain calibration, and $\mathrm{dE} / \mathrm{dx}$ measurements are discussed.

10th International Conference on Large Scale Applications and Radiation Hardness of Semiconductor Detectors,

July 6-8, 2011

Firenze Italy

\footnotetext{
*Speaker.

${ }^{\dagger}$ For the CMS Collaboration.
} 


\section{Introduction}

The Large Hadron Collider (LHC) is the largest and highest-energy particle accelerator in operation in the world and provides pp collisions to the CMS experiment at a center-of-mass energy of $\sqrt{s}=7 \mathrm{TeV}$. The CMS detector [1] is one of two general-purpose particle detectors located along the $27 \mathrm{~km}$ LHC accelerator. The CMS detector has already recorded more than $1 \mathrm{fb}^{-1}$ of data and expects to collect up to $5 \mathrm{fb}^{-1}$ of data by the end of the 2011 run.

At the center of the CMS detector are two silicon tracking devices: the silicon strip and silicon pixel detectors, collectively known as the CMS Tracker. These detectors are surrounded by electromagnetic and hadronic calorimeters and a large superconducting coil, which provides a 3.8 T magnetic field.

The strip detector is the largest silicon tracker ever constructed, covering nearly $200 \mathrm{~m}^{2}$ of active silicon surface area, with 24,244 individual silicon sensors and nearly 10 million readout channels. The strip detector is partitioned into subdetectors as follows: the tracker inner barrel (TIB) and the tracker inner disks (TID) are the structures closest to the interaction point. The TIB has 4 layers of silicon modules and the TID has three disks on either side of the interaction point. The larger tracker outer barrel (TOB) surrounds the TIB and TID and consists of 6 layers. Finally, two tracker end caps (TEC+ and TEC-) enclose the inner parts on either side of the interaction point and have nine disks each.

Stereo modules occupy the first two layers of the TIB and TOB, as well as rings 1 and 2 of the TID (1,2, and 5 in the TEC). A cross-section of one quadrant of the silicon strip tracker is displayed in Figure 1. The position of both single-sided and double-sided modules are shown. Double-sided modules are constructed of two silicon modules mounted back-to-back with a 150 mrad stereo angle, which is useful for obtaining $2 \mathrm{D}$ hit resolution.

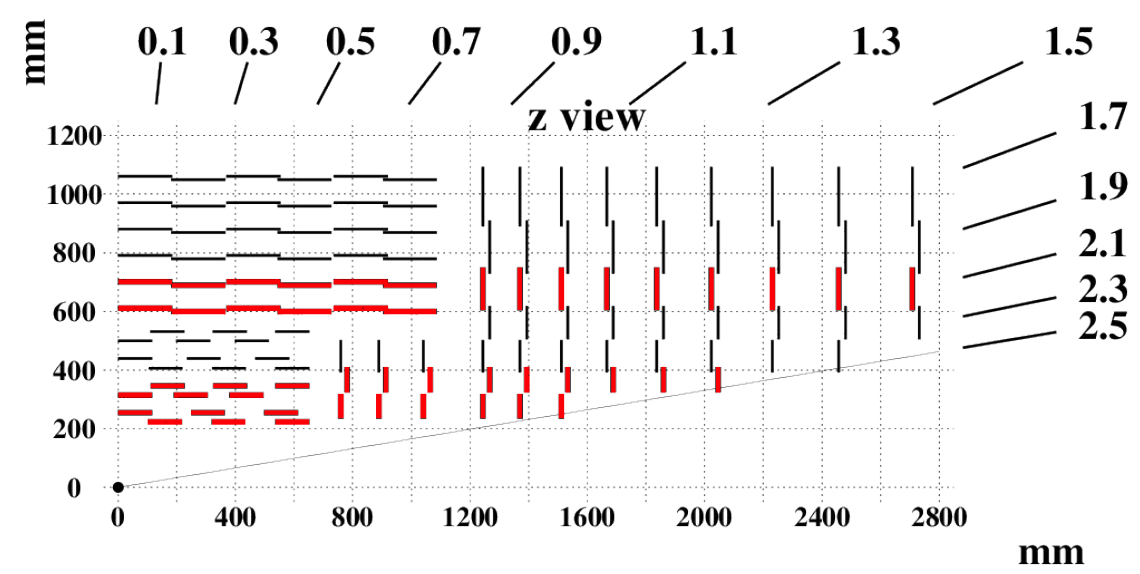

Figure 1: Cross-section of one quadrant of the CMS silicon strip detector. Positions of single-sided (black) and double-sided (red) modules are shown.

The pixel detector has an active silicon surface area of $1.1 \mathrm{~m}^{2}$ and is comprised of nearly 66 million readout channels. The pixel sensors are arranged in three barrel layers (BPIX) and two forward end-cap disks (FPIX) on either side of the interaction point. The BPIX layers are located 
at radii $\mathrm{r}=4.2,7.3$ and $11 \mathrm{~cm}$ and the FPIX disks at $\mathrm{z}=34.5$ and $46.5 \mathrm{~cm}$. These are shown in Figure 2.

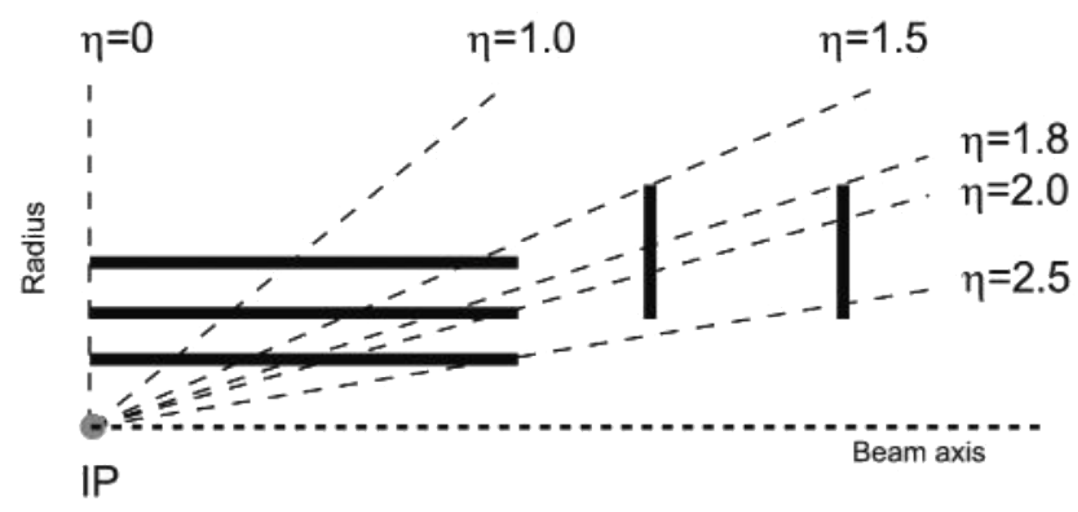

Figure 2: Cross-section of one quadrant of the CMS pixel detector.

The Tracker was designed to provide momentum resolution of $\mathrm{dp} / \mathrm{p}=10 \%$ for $1 \mathrm{TeV}$ particles, highly efficient reconstruction of charged particle tracks, excellent separation between two tracks, and radiation hardness to withstand the intense LHC luminosities. All this is done while at the same time minimizing the material budget.

\section{Status and Operation of the Tracker}

As in 2010, the CMS Tracker again operated under very stable conditions during the $2011 \mathrm{pp}$ run. The strip detector currently operates at $4^{\circ} \mathrm{C}$, while the pixel detector operates at $7.4^{\circ} \mathrm{C}$. Cooling to the strip detector is provided by $\mathrm{C}_{6} \mathrm{~F}_{14}$ along 180 cooling lines from two cooling plants. The cooling system is very reliable and the total leak rate of $\mathrm{C}_{6} \mathrm{~F}_{14}$ fluid is less than $0.6 \mathrm{~kg}$ per day. The pixel detector cooling system is also working reliably. Tracker power systems are very reliable: the strip detector exchanged less than $1 \%$ of its power supply units during the first half of 2011. DAQ electronics are robust for both strip and pixel detectors; only minor firmware adjustments were needed to fix event handling and to cope with high event multiplicity. The Detector Control System (DCS), providing power status, temperature and relative humidity monitoring of the Tracker, is reliable, as well is the Data Quality Monitoring (DQM) service. Collectively, the Tracker has provided greater than $98 \%$ uptime to CMS during the 2010 and 2011 runs.

The overall fraction of alive strip detector channels is $97.75 \%$. The fractions broken down by the difference partition are listed in Table 1. A map showing the inactive channels is presented in Figure 3, where components that were never commissioned and are inactive are shown in white, components which became inactive during operation are shown in red, and components that are active are shown in green. Inactive channels can generally be grouped into three categories depending on the severity of their failure mode: "dead" meaning permanent failure, "excluded" meaning the device occasionally fails, and "readout errors" meaning the device can be readout, but the data are not useful. The majority of inactive components that became inactive during operation are due to isolated problems in the control ring communication paths and high voltage shortages. 
Table 1: Fraction of alive silicon strip detector channels by partition.

\begin{tabular}{|l|r|r|}
\hline Partition & Percentage & Total Modules \\
\hline \hline TIB/TID & 94.3 & 3540 \\
TOB & 98.1 & 5208 \\
TEC- & 98.9 & 3200 \\
TEC+ & 98.2 & 3200 \\
\hline & 97.75 & 15148 \\
\hline
\end{tabular}

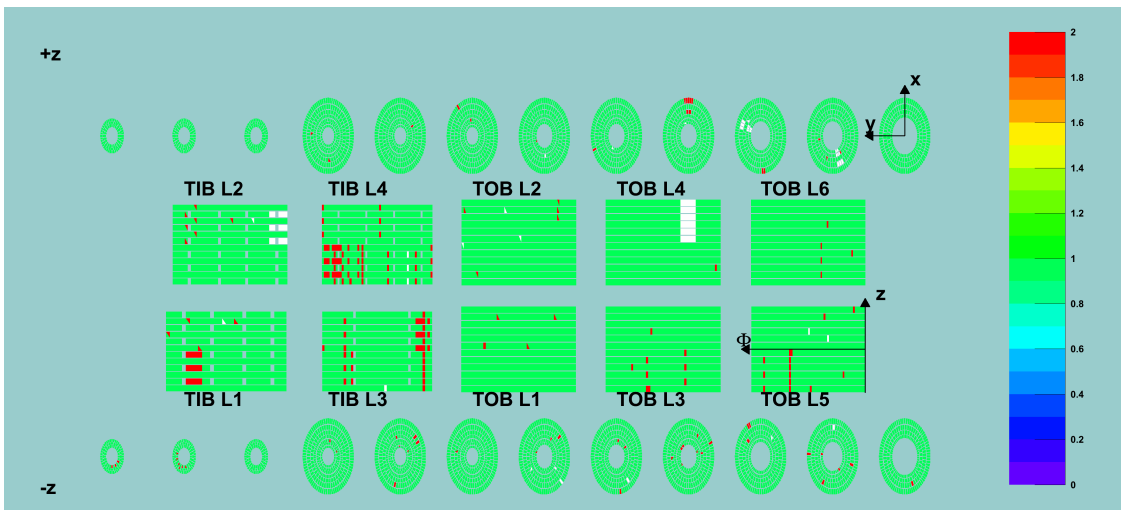

Figure 3: Device mapping of the silicon strip detector. Components that were never commissioned and are inactive are shown in white. Components that became inactive during operation are shown in red. Alive components are shown in green.

The overall fraction of alive pixel detector channels is $96.9 \%$ (FPix 92.8\%, BPix 98.4\%). The largest source of failure occurred in a single point loss in service electronics due to a failed and nonrepairable laser driver. A $0.5 \%$ per year loss in BPix channels is also observed and is consistent with failing wire bonds.

At the time of this conference, the CMS data-taking efficiency was $93 \%$ in the $2011 \mathrm{pp}$ running period. Of the $9 \%$ total CMS down-time, the silicon strip tracker accounted for $19 \%$ due to problems with the DAQ system, and $3 \%$ were due to problems with the power system. The pixel DAQ accounted for $8 \%$, while the pixel power system accounted for only $1 \%$.

\section{Calibration}

The silicon strip readout system was fully commissioned and calibrated in 2009 and partially again for the TIB partition only in 2010. Once properly calibrated, the majority of the calibration parameters remain largely unchanged until there is a non-negligible modification to the detector conditions. For example, a change to the detector hardware would require a re-calibration for those affected devices. Also, a change to the detector operating temperature, or accumulated radiation damage, may necessitate a partial or complete detector re-calibration. Radiation damage to the silicon sensors already became visible during the 2011 run [2], however the Tracker is expected to run well with the present operating conditions for the duration of the 2011 and 2012 runs. Some parameters require more regular re-calibration. The pedestal and noise values, for example, are 
needed to perform the zero-suppression and cluster finding and are updated more regularly. The calibration procedures for the strip detector are described in detail elsewhere [3].

It is necessary to optimize the sampling time of the strip detector readout at regular intervals to be in the phase with the LHC bunch crossing. This is achieved using data recorded during specialized runs using LHC collisions. These timing measurements were performed in 2009, 2010, and again repeated in March 2011 with collisions at $7 \mathrm{TeV}$.

For this measurement, the timing of each module is varied randomly around the nominal sampling time. Then the reconstructed on-track signals are studied at different delay settings. The results of this timing measurement using the March 2011 data are shown in Figure 4, where the expected $12 \mathrm{~ns}$ deconvolution pulse shape is reconstructed separately for each partition. Each pulse shape maximum should be centered at the zero offset delay bin with respect to the nominal sampling time. As shown, this was found to be the case to within $1 \mathrm{~ns}$. This minor shift did not affect the quality of data collected or the the signal-to-noise ration of the strip detector. The signal height is dependent on both the thickness of the silicon sensors and sensor orientation. The TIB partition, for example, has the thinnest sensors $(300 \mu \mathrm{m})$ which are optimized for perpendicular incident particles and has the lowest signal, whereas the $500 \mu \mathrm{m}$ TEC "thick" sensors are not optimized and have the highest signal.

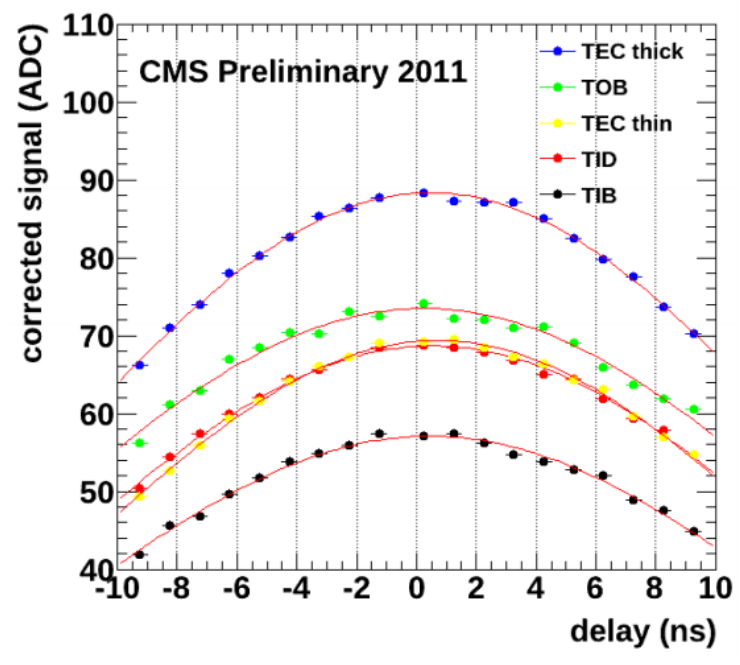

Figure 4: Timing measurement performed for each strip detector partition with collisions at $7 \mathrm{TeV}$.

A similar procedure was performed for the pixel detector where instead the on-track cluster size is optimized. The results of this scan are shown in Figure 5. Average cluster size (shown in black) and efficiency (shown in red) are plotted versus the relative phase between the LHC clock and the pixel clock. The clock phase of the pixel detector was chosen to be 13 ns. The operating point is well within the maximum efficiency plateau.

\section{Energy deposits in Silicon}

Measurements of the energy deposited versus silicon thickness ( $\mathrm{dE} / \mathrm{dx}$ ) were also made using collisions at $7 \mathrm{TeV}$ during the 2011 run. These measurements are useful for identifying charged 


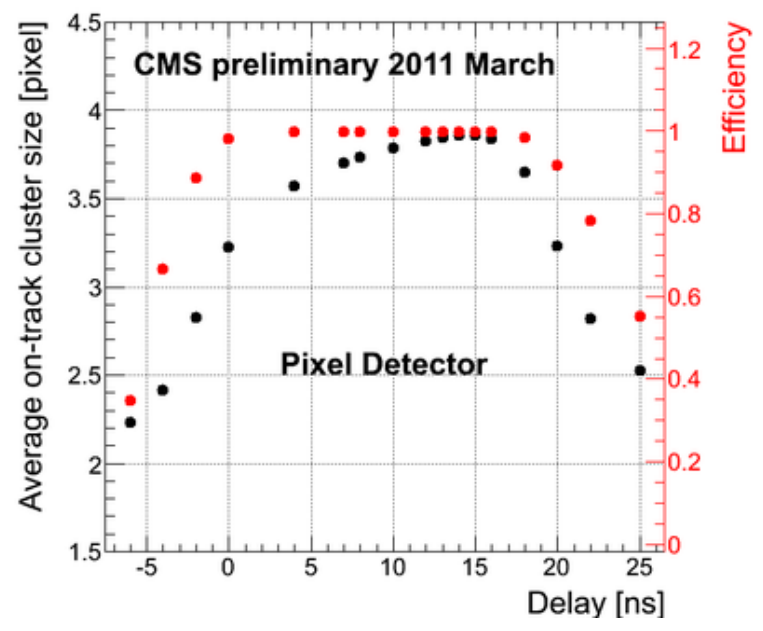

Figure 5: Timing measurement performed for the pixel detector with collisions at $7 \mathrm{TeV}$.

particles in the silicon detector. Figure 6 shows $\mathrm{dE} / \mathrm{dx}$ as measured in the silicon strip tracker versus the track momentum. Kaons, protons, deuterons and tritium are clearly visible in this plot. The red lines are the expected curves from the Bethe-Bloch formula, extrapolated from a fit of the proton line. A small deviation is observed at large $\mathrm{dE} / \mathrm{dx}$ values.

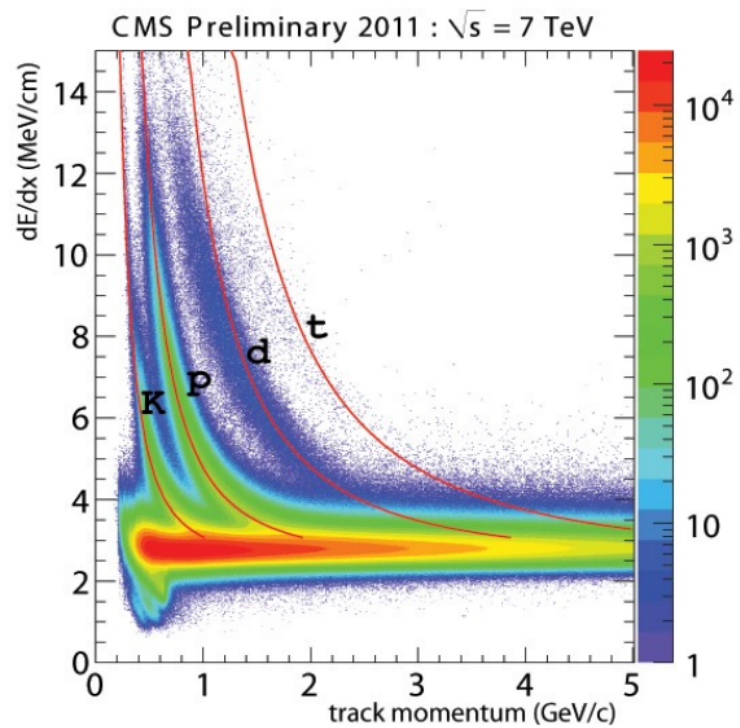

Figure 6: Measured dE/dx in the silicon strip detector versus track momentum. Expected curves from the Bethe-Bloch formula are shown in red.

\section{Conclusions}

The CMS silicon Tracker is in excellent shape after more than two years of operations. The overall detector alive fraction is nearly $98 \%$ for the strip detector and $97 \%$ for the pixel detector. 
Environmental, power, data acquisition, and monitoring services are providing robust and stable functionality and contribute to the excellent data collection efficiency obtained by CMS during the 2011 pp run at $\sqrt{s}=7 \mathrm{TeV}$ collisions. The LHC delivered already $1 \mathrm{fb}^{-1}$ of data to CMS, of which $93 \%$ was successfully recorded, and up to $5 \mathrm{fb}^{-1}$ of data are expected by the end of the 2011 run.

\section{References}

[1] S. Chatrchyan et al. [CMS Collaboration], "The CMS experiment at the CERN LHC," JINST 3 (2008) S08004.

[2] C. Barth et al. [CMS Collaboration], "Evolution of silicon sensors characteristics of the current CMS tracker" Nuclear Instruments and Methods in Physics Research A, 658, 6-10, (2011).

[3] E. Butz et al. [CMS Collaboration], "CMS Silicon Strips Operations and Performance" Proceedings of Science, Vertex (2010). 